\title{
Reconstruction-Based 3D/2D Image Registration
}

\author{
Dejan Tomaževič, Boštjan Likar, and Franjo Pernuš \\ University of Ljubljana, Faculty of Electrical Engineering, \\ Tržaška 25, 1000 Ljubljana, Slovenia \\ \{dejan.tomazevic, bostjan.likar, franjo.pernus\}@fe.uni-lj.si
}

\begin{abstract}
In this paper we present a novel 3D/2D registration method, where first, a 3D image is reconstructed from a few 2D X-ray images and next, the preoperative 3D image is brought into the best possible spatial correspondence with the reconstructed image by optimizing a similarity measure. Because the quality of the reconstructed image is generally low, we introduce a novel asymmetric mutual information similarity measure, which is able to cope with low image quality as well as with different imaging modalities. The novel $3 \mathrm{D} / 2 \mathrm{D}$ registration method has been evaluated using standardized evaluation methodology and publicly available 3D CT, 3DRX, and MR and 2D X-ray images of two spine phantoms [1], for which gold standard registrations were known. In terms of robustness, reliability and capture range the proposed method outperformed the gradient-based method [2] and the method based on digitally reconstructed radiographs (DRRs).
\end{abstract}

\section{Introduction}

In image-guided therapy, preoperative three-dimensional (3D) computed tomography (CT) or magnetic resonance (MR) images and models of anatomical structures, obtained by image segmentation, serve for preoperative planning and simulation, and as "background" shown on the monitor in the treatment room onto which models of surgical instruments or of radiation beams are projected. The link between a preoperative 3D image and intraoperative physical space of the patient is established by registration of the preoperative image either directly to the patient or to intraoperative images of the patient. When two-dimensional (2D) images are acquired intraoperatively the pre- to intraoperative image registration is called $3 \mathrm{D} / 2 \mathrm{D}$ registration. In the last decade, different 3D/2D image registration methods have been proposed. The segmentation-based 3D/2D registration methods [3-6] minimize the spatial distance between positions of corresponding geometrical features that have previously been extracted from pre- and intraoperative images. The drawback of these methods is that intraoperative segmentation errors propagate to errors in registration. Intensity-based 3D/2D registration methods [7-10] rely on image intensities or intensity gradients of pixels and voxels. The most popular intensity-based 3D/2D registration method optimizes the similarity measure calculated from overlapping CT-based digitally reconstructed radiographs (DRRs) and X-ray images [7-10]. Intensity-based 3D/2D registration methods are considered to be more accurate than segmentation-based methods but slower due to time consuming calculation of DRRs. Hybrid methods combine elements of segmentation- and intensity-based methods with the purpose to achieve 
the speed of segmentation-based methods and the accuracy of intensity-based methods $[2,11,12]$. From validation results provided by the authors of intensity-based and hybrid methods and from a recent comparison study of van de Kraats et al. [1], it is obvious that some of these methods are highly accurate when registering a CT image to two or more X-ray images. However, their capture ranges are rather small and they are not robust enough. Besides, 3D/2D registration of $\mathrm{MR}$ to X-ray images remains a challenging problem.

In this paper we propose a novel 3D/2D image registration method which first reconstructs a 3D image from a few 2D X-ray images and then matches this image to either a CT, MR, or 3DRX pre-operative image. The quality of a 3D image, reconstructed from a small number of 2D images will definitively be low. The similarity measure applied in such a registration should therefore be able to cope, among others, with low image quality of one image as well as with differences in imaging modalities. For this purpose we introduce a novel and powerful similarity measure, which we call asymmetric multi-feature mutual information measure. The measure is based on the multi-feature mutual information measure, recently proposed by Tomaževič at al. [13].

\section{Asymmetric Multi-feature Mutual Information}

Let the two images to be registered be denoted as floating image $A$ and reference image $B$ and represented by vector functions $\mathbf{z}_{a}(x)$ and $\mathbf{z}_{b}(x)$ of position $x$ in image space, respectively. Each vector function $\mathbf{z}(x)$ is comprised of values of $K$ image features, $\mathbf{z}(x)=\left(\mathrm{z}_{1}(x), \ldots, \mathrm{z}_{K}(x)\right)$. Let, for a given spatial transformation $T, S M$ denote the similarity measure between corresponding feature sets $\mathbf{z}_{a}(x)$ and $\mathbf{z}_{b}(T(x))$. Registration seeks the spatial transformation $\hat{T}$ that maximizes $S M$

$$
\hat{T}=\underset{T}{\arg \max } \operatorname{SM}\left(\mathbf{z}_{a}(x), \mathbf{z}_{b}(T(x))\right) .
$$

Let the values of $\mathbf{z}_{a}(x)$ and $\mathbf{z}_{b}(x)$ be the observed values of vectors of random variables $\mathbf{Z}_{a}$ and $\mathbf{Z}_{b}$, respectively. In terms of entropy, multi-feature mutual information (MMI) [13], which represents a generalization of the widely used single-feature mutual information criteria $[14,15]$, is defined as

$$
\operatorname{MMI}\left(\mathbf{Z}_{a}, \mathbf{Z}_{b}\right)=H\left(\mathbf{Z}_{a}\right)+H\left(\mathbf{Z}_{b}\right)-H\left(\mathbf{Z}_{a}, \mathbf{Z}_{b}\right)
$$

where $H\left(\mathbf{Z}_{a}\right), H\left(\mathbf{Z}_{b}\right)$ and $H\left(\mathbf{Z}_{a}, \mathbf{Z}_{b}\right)$ are entropies of vectors of random variables $\mathbf{Z}_{a}=\left(Z_{a 1}, \ldots, Z_{a K}\right), \mathbf{Z}_{b}=\left(Z_{b 1}, \ldots, Z_{b K}\right)$ and $\left(\mathbf{Z}_{a}, \mathbf{Z}_{b}\right)=\left(Z_{a 1}, \ldots, Z_{a K}, Z_{b 1}, \ldots, Z_{b K}\right)$, respectively. In general, entropy of a $K$-dimensional random variable $\mathbf{Z}, \mathbf{Z}=\left(Z_{1}, \ldots, Z_{K}\right)$ is defined as

$$
H(\mathbf{Z})=-\int p(\mathbf{z}) \log p(\mathbf{z}) d \mathbf{z}=-\int \ldots \int p\left(z_{1}, \ldots, z_{K}\right) \log p\left(z_{1}, \ldots, z_{K}\right) d z_{1} \ldots d z_{K} .
$$

In case $Z_{1}, \ldots, Z_{K}$ are discrete random variables, entropy $H(\mathbf{Z})$ is obtained as

$$
H(\mathbf{Z})=-\sum_{\mathbf{z}} p(\mathbf{z}) \log p(\mathbf{z})=-\sum_{z_{1}} \ldots \sum_{z_{K}} p\left(z_{1}, \ldots, z_{K}\right) \log p\left(z_{1}, \ldots, z_{K}\right) .
$$


As for single-feature mutual information, the multivariate probability distributions $p\left(\mathbf{z}_{a}\right), p\left(\mathbf{z}_{b}\right)$ and $p\left(\mathbf{z}_{a}, \mathbf{z}_{b}\right)$ can be estimated from joint histograms [16]. Unfortunately, even in case of two features, the four-dimensional histogram $h\left(z_{a}, \mathrm{z}_{b}\right)$, will probably be so sparse that a meaningful estimation of $p\left(\mathbf{z}_{a}, \mathbf{z}_{b}\right)$ will become practically impossible. For this reason, Tomaževič et al. [13] proposed to decompose the floating and the reference image features into a basic feature $i(x)$ and additional features $\mathbf{v}(x)$, i.e. $\mathbf{z}_{a}(x)=\left(i_{a}(x), \mathbf{v}_{a}(x)\right)$ and $\mathbf{z}_{b}(x)=\left(i_{b}(x), \mathbf{v}_{b}(x)\right)$. For the purpose of registering a preoperative image to a reconstructed image we propose that only one vector function, say $\mathbf{z}_{a}(x)$, is divided, resulting in $\mathbf{z}_{a}(x)=\left(i_{a}(x), \mathbf{v}_{a}(x)\right)$. Using the known property of entropy [17] that

$$
H(\mathbf{Z})=H\left(Z_{k}\right)+H\left(\left(Z_{1}, \ldots, Z_{k-1}, Z_{k+1}, \ldots, Z_{K}\right) \mid Z_{k}\right),
$$

MMI is obtained as

$$
\begin{aligned}
\operatorname{MMI}\left(\mathbf{Z}_{a},\right. & \left.\mathbf{Z}_{b}\right)=H\left(\mathbf{Z}_{a}\right)+H\left(\mathbf{Z}_{b}\right)-H\left(\mathbf{Z}_{a}, \mathbf{Z}_{b}\right)= \\
& =H\left(I_{a}, \mathbf{V}_{a}\right)+H\left(\mathbf{Z}_{b}\right)-H\left(I_{a}, \mathbf{V}_{a}, \mathbf{Z}_{b}\right)= \\
& =H\left(I_{a}\right)+H\left(\mathbf{V}_{a} \mid I_{a}\right)+H\left(\mathbf{Z}_{b}\right)-H\left(I_{a}\right)-H\left(\mathbf{V}_{a}, \mathbf{Z}_{b} \mid I_{a}\right)= \\
& =H\left(\mathbf{Z}_{b}\right)+H\left(\mathbf{V}_{a} \mid I_{a}\right)-H\left(\mathbf{V}_{a}, \mathbf{Z}_{b} \mid I_{a}\right)
\end{aligned}
$$

where $H\left(\mathbf{Z}_{b}\right)$ is the entropy of $\mathbf{Z}_{b}$, and $H\left(\mathbf{V}_{a} \mid I_{a}\right)$ and $H\left(\mathbf{V}_{a}, \mathbf{Z}_{b} \mid I_{a}\right)$ are entropies of $\mathbf{V}_{a}$ and $\mathbf{V}_{a} \mathbf{Z}_{b}$ under the condition $I_{a}$, respectively. We call this similarity measure the asymmetric MMI (AMMI). Assuming that distributions of $\mathbf{Z}_{b}, \mathbf{V}_{a} \mid i_{a}$ and $\mathbf{V}_{a} \mathbf{Z}_{b} \mid i_{a}$ are normal, and knowing the distribution $p\left(i_{a}\right)$ and covariance matrices $\boldsymbol{\Sigma}_{\mathbf{z}_{b}}, \boldsymbol{\Sigma}_{\mathbf{v}_{a} \mid i_{a}}$ and $\boldsymbol{\Sigma}_{\mathbf{V}_{a} \mathbf{Z} b \mid i_{a}}$, entropy $H\left(\mathbf{Z}_{b}\right)$ and conditional entropies $H\left(\mathbf{V}_{a} \mid I_{a}\right)$ and $H\left(\mathbf{V}_{a}, \mathbf{Z}_{b} \mid I_{a}\right)$ may be defined by

$$
\begin{gathered}
H(\mathbf{Y} \mid I)=\sum_{i} p(i) H(\mathbf{Y} \mid i), \\
H(\mathbf{Y})=\frac{1}{2} \log \left|\Sigma_{\mathbf{y}}\right|+\frac{n}{2} \log (2 \pi e)
\end{gathered}
$$

Covariance matrices $\boldsymbol{\Sigma}_{\mathbf{v} a \mid i_{a}}$ and $\Sigma_{\mathbf{v}_{a} \mathbf{Z} b \mid i_{a}}$, are estimated for every feature value $i_{a}$. This approach requires much fewer samples than the estimation through high dimensional histograms. Moreover, in the case of AMMI, a one-dimensional histogram $h\left(i_{a}\right)$ is needed to estimate the probability distribution of the basic feature. With a onedimensional histogram more samples become available for estimation of an individual covariance matrix. Entropies $H\left(\mathbf{Z}_{b}\right), H\left(\mathbf{V}_{a} \mid I_{a}\right)$ and $H\left(\mathbf{V}_{a}, \mathbf{Z}_{b} \mid I_{a}\right)$, estimated by Eqs. 7 and 8 are monotonically increasing functions of variances of random variables $\mathbf{Z}_{b}, \mathbf{V}_{a} \mid i_{a}$ and $\mathbf{V}_{a} \mathbf{Z}_{b} \mid i_{a}$, respectively. The condition that random variable $\mathbf{Z}_{b}$, and conditional random variables $\mathbf{V}_{a} \mid i_{a}$ and $\mathbf{V}_{a} \mathbf{Z}_{b} \mid i_{a}$ are normally distributed will, generally, not be fulfilled in practice. Nevertheless, Eqs. 7 and 8 may be used to estimate entropies needed to calculate AMMI as long as the real, but unknown entropies are also monotonically increasing functions of variances of $\mathbf{Z}_{b}, \mathbf{V}_{a} \mid i_{a}$ and $\mathbf{V}_{a} \mathbf{Z}_{b} \mid i_{a}$.

\section{Experiments}

The proposed method has been evaluated and compared to the gradient-based method of Tomaževič at al. [2] using publicly available image data [1]. The image dataset 
comprised 2D fluoroscopic X-ray images and 3D 3DRX, CT and MR images of two defrosted segments of a vertebral column. The first vertebral column consisted of three thoracolumbar vertebrae bodies while the second segment comprised five thoracic vertebrae bodies. Some soft tissue was still present around both segments. The 2D fluoroscopic images were obtained with a clinical 3DRX system (Integris BV5000, Philiphs Medical System, Best, The Netherlands). A set of 100 X-ray images was acquired for each spinal segment in 8 seconds run of 180 degrees rotation around the imaged object. For each vertebral column, a 3DRX image was reconstructed from a set of $100 \mathrm{X}$-ray images using a filtered back-projection reconstruction technique [18]. The two CT-images were acquired with a clinical 16-detectorrow multi-slice CT scanner (MSCT, Philips Medical System, Best, The Netherlands). The MR images were obtained with a clinical 1.5 Tesla MR scanner (Gyroscan NT, Philips Medical System, Best, The Netherlands) using a sagittal 3D turbo spin echo acquisition and turbo factor of 29, TR/TE of $1500 \mathrm{~ms} / 90 \mathrm{~ms}$. The MR images were corrected with the retrospective intensity inhomogeneity correction method based on information minimization [19]. The ground truth registration between 3DRX images and $2 \mathrm{D}$ projection images was established in the process of creating 3DRX images, while the gold-standard registration between CT and MR images with 2D fluoroscopic images was obtained by $3 \mathrm{D} / 3 \mathrm{D}$ rigid registration of $\mathrm{CT}$ and $\mathrm{MR}$ images to corresponding 3DRX images using the mutual information maximization registration method [16]. Eight volumes of interest (VOIs), each containing a whole vertebra body and less than a quarter of neighboring vertebrae, were manually determined on 3DRX image volumes. VOIs in CT and MR images corresponding to 3DRX VOIs were defined by the gold standard registration. Examples of fluoroscopic, 3DRX, CT, and MR images of a VOI are shown in Fig. 1.
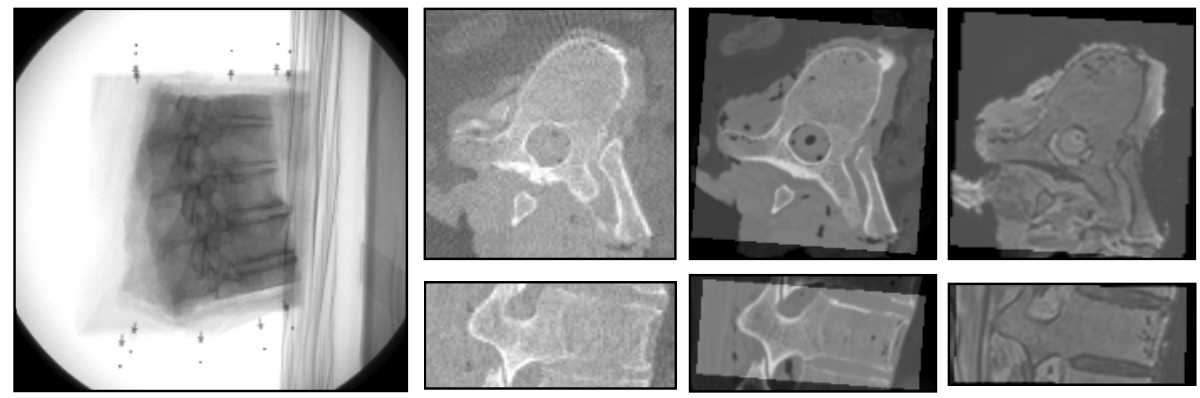

Fig. 1. Fluoroscopic (left) and transversal (top row) and lateral planes (bottom row) of corresponding VOIs taken from 3DRX (second column), CT (third column) and MR volumes (right column)

The gradient-based method was implemented as in [2]. The 2D X-ray images were blurred with a Gaussian kernel of $0.5 \mathrm{~mm}$, the 3D images were isotropically resampled to $0.96 \mathrm{~mm}$ voxel sizes by linear interpolation, while the threshold to extract bone edges from VOIs was set to 18 for 3DRX and CT images and 15 for MR images. 3DRX and CT VOIs were registered to $3 \mathrm{X}$-ray images while MR VOIs were registered to $11 \mathrm{X}$-ray images because of the larger difference in modalities. The 
angle between each of the 3 (11) image views was approximately $60^{\circ}\left(15^{\circ}\right)$. For the novel method, the 3D image of a whole spinal segment was reconstructed from the same 3 and 11 X-ray images by the SART reconstruction method [20]. The sizes of reconstructed 3D images were 128x128x128 and 128x188x128 image elements for the first and second spinal segment, respectively, with isotropic spatial resolution of $0.63 \mathrm{~mm}$. The preoperative 3D image was taken as the floating image $A$ and the reconstructed image as the reference image $B$. Both feature sets $\mathbf{z}_{a}(x)$ and $\mathbf{z}_{b}(x)$, characterizing the preoperative and reconstructed image, respectively, consisted of image intensity $i(x)$ and image intensity gradient $\mathbf{v}(x)$ features, i.e. $\mathbf{z}_{a}(x)=\left(i_{a}(x), \mathbf{v}_{a}(x)\right)$ and $\mathbf{z}_{b}(x)=\left(i_{b}(x), \mathbf{v}_{b}(x)\right)$. To reduce sensitivity to image noise and non-isotropic image acquisition, the gradients of all 3D images were obtained after convolving 3D intensities with a Gaussian. Kernel scales of $0.5 \mathrm{~mm}$ and $0.35 \mathrm{~mm}$ were applied to the original 3D images and 3D reconstructed images, respectively. The AMMI similarity measure (Eq. 6) was used to measure the match between $\mathbf{z}_{a}(x)$ and $\mathbf{z}_{b}(T(x))$. Image intensity of the preoperative (floating) image $i_{a}(x)$ was the only feature whose probability distribution was estimated by using one-dimensional histogram $h\left(i_{a}\right)$ of intensity values. A histogram, having 64 bins was used to assure statistical power. Assuming normal distribution of $\mathbf{Z}_{b}, \mathbf{V}_{a} \mid i_{a}$ and $\mathbf{V}_{a} \mathbf{Z}_{b} \mid i_{a}$, the multivariate probability distribution $p\left(\mathbf{z}_{b}\right)$ was estimated through the covariance matrix $\Sigma_{\mathbf{z} b}$, while the distributions $p\left(\mathbf{v}_{a} \mid i_{a}\right)$ and $p\left(\mathbf{v}_{a}, \mathbf{z}_{b} \mid i_{a}\right)$ were estimated through conditional covariance matrices $\boldsymbol{\Sigma}_{\mathbf{v}_{a l i} i_{a}}$ and $\boldsymbol{\Sigma}_{\mathbf{v}_{a} \mathbf{Z}_{b} \mid i_{a}}$, respectively, for every intensity value $i_{a}$. Powell's optimization method [21] was used in both methods to optimize the given similarity measure for six rigid-body transformation parameters $\left(t_{x}, t_{y}, t_{z}, \omega_{x}, \omega_{y}, \omega_{z}\right)$.

Both $3 \mathrm{D} / 2 \mathrm{D}$ registration methods were evaluated using the standardized evaluation methodology of van de Kraats et al. [1]. The evaluation methodology uses the mean target registration error (mTRE) to measure the distance of a VOI position from the gold standard before and after registration. The positions of all image elements in a VOI were used as target points. For evaluating the capture range and robustness of a 3D/2D registration method, van de Kraats et al. [1] provided 200 starting positions for each VOI. The 200 starting position were randomly generated around the gold standard position in such a way that the distance from gold standard measured by mTRE was uniformly distributed in the interval of 0-20 mm. For each of 3DRX, CT or MR modalities 1600 registrations to X-ray images (reconstructed images) were thus performed, 200 per each of the 8 VOIs. Each registration was considered successful, if mTRE after registration was lower than $2 \mathrm{~mm}$. The registration error was defined as mTRE of all successful registrations, while the capture range was defined as the distance from gold standard for which the registration had proved to be successful in at least $95 \%$ of all cases.

\section{Results and Conclusion}

The registration errors, capture ranges and percentages of successful registrations for both methods and different modalities are shown in Table1. The novel method is a little less accurate, has a somewhat larger capture range, especially for MR to X-ray registrations and is much more robust that the gradient-based method. Fig. 2 shows 
Table 1. Mean TREs, capture ranges and percentage of successful registrations for the gradient-based (GBM) and reconstruction-based (RBM) methods

\begin{tabular}{|l|l|cc|cc|cc|}
\hline Modality & Views & \multicolumn{2}{|c|}{ mTRE (mm) } & \multicolumn{2}{|c|}{$\begin{array}{c}\text { Capturing } \\
\text { range (mm) }\end{array}$} & \multicolumn{2}{|c|}{$\begin{array}{c}\text { Successful } \\
\text { registrations } \\
(\boldsymbol{\%})\end{array}$} \\
\cline { 3 - 8 } & & GBM & RBM & GBM & RBM & GBM & RBM \\
\hline 3DRX & $\mathbf{3}$ & 0.19 & 0.33 & 7 & 9 & $68 \%$ & $89 \%$ \\
CT & $\mathbf{3}$ & 0.32 & 0.37 & 7 & 7 & $63 \%$ & $78 \%$ \\
MR & $\mathbf{1 1}$ & 0.50 & 0.67 & 2 & 7 & $23 \%$ & $84 \%$ \\
\hline
\end{tabular}
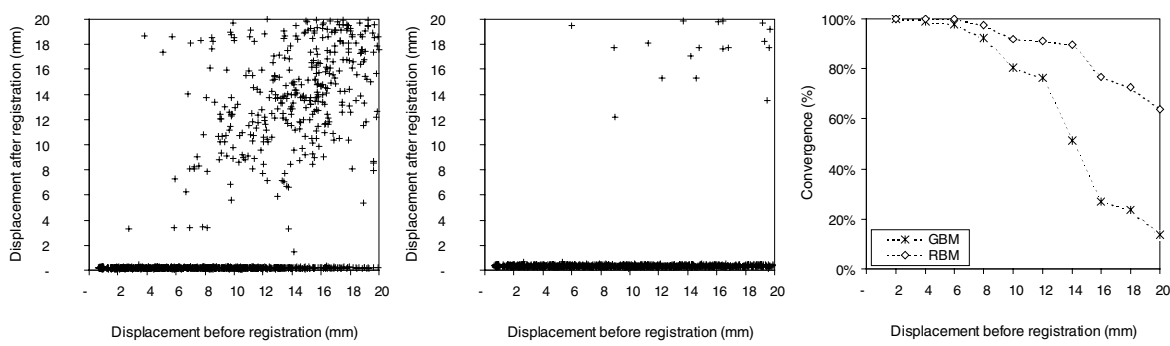

Displacement before registration $(\mathrm{mm})$

Displacement before registration $(\mathrm{mm})$
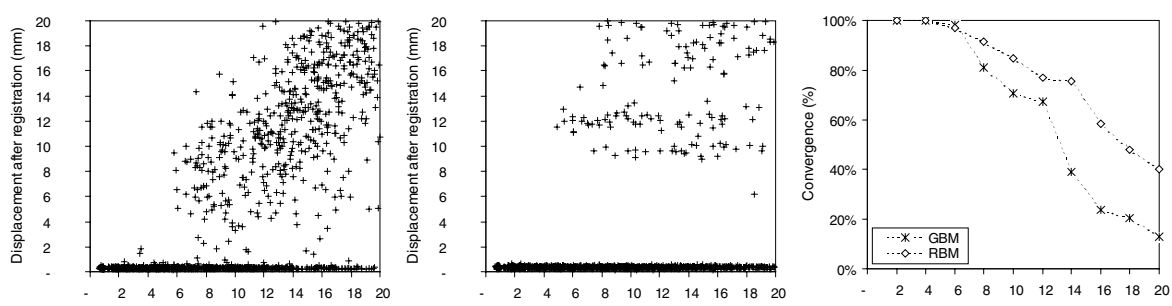

Displacement before registration $(\mathrm{mm})$

Displacement before registration $(\mathrm{mm})$

Displacement before registration $(\mathrm{mm})$
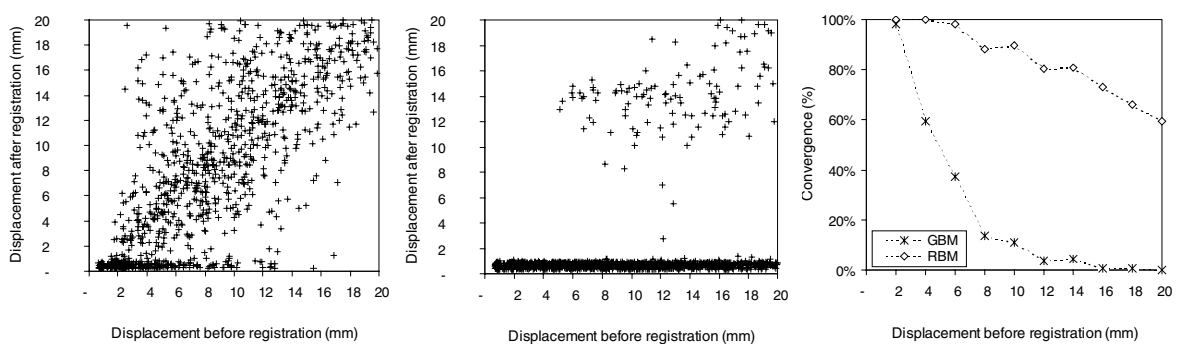

Fig. 2. Registration results of 3DRX (first row) and CT VOIs (second row) to $3 \mathrm{X}$-ray images and MR VOIs to $11 \mathrm{X}$-ray images (third rows). Scatter diagrams of displacements before and after registration for the gradient-based method (GBM) (left column) and the reconstruction based method (RBM) (middle column), and the proportions of correct registrations (right column). 
the results in more detail. The results are in the form of scatter diagrams of displacements (mTRE) before and after registration and proportions of successful registrations (convergence) with respect to the initial displacement. For all modalities the proposed reconstruction-based method was successful in a significantly larger number of registrations than the gradient-based method. As expected, the proportion of successful registrations fell with the extent of initial displacement.

The proposed novel approach to $3 \mathrm{D} / 2 \mathrm{D}$ registration based on $3 \mathrm{D}$ integration of $2 \mathrm{D}$ information is general and does not make any constraints on the modalities and anatomies involved in the registration. The experimental results show that the proposed method outperforms the gradient-based method with respect to capture range and proportion of correct registrations.

\section{Acknowledgements}

The authors would like to thank Dr. R.L.A.W. Bleys from the Anatomy department at the University Medical Center Utrecht for making the spinal segments available and E.B. van de Kraats from Image Sciences Institute, Utrecht, for acquiring and processing image data.

\section{References}

1. E. B. van de Kraats, G. P. Penney, D. Tomaževič, T. van Walsum, and W. J. Niessen, "Standardized Evaluation of 2D-3D Registration," in Lecture Notes in Computer Science, vol. 3216, Medical Image Computing and Computer-Assisted Intervention - MICCAI 2004, C. Barillot, D. R. Haynor, and P. Hellier, Eds., 2004, pp. 574-581.

2. D. Tomaževič, B. Likar, T. Slivnik, and F. Pernuš, "3-D/2-D registration of CT and MR to X-ray images," IEEE Trans Med Imaging, vol. 22, pp. 1407-16, 2003.

3. J. Feldmar, N. Ayache, and F. Betting, "3D-2D projective registration of free-form curves and surfaces," Computer Vision and Image Understanding, vol. 65, pp. 403-424, 1997.

4. A. Gueziec, P. Kazanzides, B. Williamson, and R. H. Taylor, "Anatomy-based registration of CT-scan and intraoperative X-ray images for guiding a surgical robot," IEEE Trans Med Imaging, vol. 17, pp. 715-728, 1998.

5. A. Gueziec, K. Wu, A. Kalvin, B. Williamson, P. Kazanzides, and R. Van Vorhis, "Providing visual information to validate 2-D to 3-D registration," Med Image Anal, vol. 4, pp. 357-74, 2000.

6. S. Lavallee and R. Szeliski, "Recovering the Position and Orientation of Free-Form Objects from Image Contours Using 3d Distance Maps," IEEE Transactions on Pattern Analysis and Machine Intelligence, vol. 17, pp. 378-390, 1995.

7. L. Lemieux, R. Jagoe, D. R. Fish, N. D. Kitchen, and D. G. Thomas, "A patient-tocomputed-tomography image registration method based on digitally reconstructed radiographs," Med Phys, vol. 21, pp. 1749-60, 1994.

8. G. P. Penney, J. Weese, J. A. Little, P. Desmedt, D. L. Hill, and D. J. Hawkes, "A comparison of similarity measures for use in 2-D-3-D medical image registration," IEEE Trans Med Imaging, vol. 17, pp. 586-595, 1998.

9. J. H. Hipwell, G. P. Penney, R. A. McLaughlin, K. Rhode, P. Summers, T. C. Cox, J. V. Byrne, J. A. Noble, and D. J. Hawkes, "Intensity-based 2-D-3-D registration of cerebral angiograms," IEEE Trans Med Imaging, vol. 22, pp. 1417-26, 2003. 
10. J. Weese, G. P. Penney, P. Desmedt, T. M. Buzug, D. L. Hill, and D. J. Hawkes, "Voxelbased 2-D/3-D registration of fluoroscopy images and CT scans for image-guided surgery," IEEE Trans Inf Technol Biomed, vol. 1, pp. 284-93, 1997.

11. K. G. Gilhuijs, P. J. van de Ven, and M. van Herk, "Automatic three-dimensional inspection of patient setup in radiation therapy using portal images, simulator images, and computed tomography data," Med Phys, vol. 23, pp. 389-99, 1996.

12. H. Livyatan, Z. Yaniv, and L. Joskowicz, "Gradient-based 2-D/3-D rigid registration of fluoroscopic X-ray to CT," IEEE Trans Med Imaging, vol. 22, pp. 1395-406, 2003.

13. D. Tomaževič, B. Likar, and F. Pernuš, "Multi-feature mutual information," in Medical Imaging: Image Processing, vol. 5370, J. M. Fitzpatrick and M. Sonka, Eds. Bellingham, WA: SPIE, 2004, pp. 143-154.

14. P. Viola and W. M. Wells, 3rd, "Alignment by maximization of mutual information," in Proc. 5th Int. Conf. Computer Vision. Boston, MA, 1995, pp. 16-23.

15. A. Collignon, F. Maes, D. Delaere, D. Vandermeulen, P. Suetens, and G. Marchal, "Automated multi-modality image registration based on information theory," in Information Processing in Medical Imaging. Norwell, MA: Kluwer, 1995, pp. 263-274.

16. F. Maes, A. Collignon, D. Vandermeulen, G. Marchal, and P. Suetens, "Multimodality image registration by maximization of mutual information," IEEE Trans Med Imaging, vol. 16, pp. 187-98, 1997.

17. T. M. Cover and J. A. Thomas, Elements of Information Theory. New York, USA: John Wiley \& Sons, 1991.

18. M. Grass, R. Koppe, E. Klotz, R. Proksa, M. Kuhn, H. Aerst, and J. O. d. B. Beek, "Threedimensional reconstruction of high contrast objects using C-arm image intensifier projection data," Computerized Medical Imaging and Graphics, vol. 23, pp. 311-321, 1999.

19. B. Likar, M. A. Viergever, and F. Pernuš, "Retrospective correction of MR intensity inhomogeneity by information minimization," IEEE Trans Med Imaging, vol. 20, pp. 1398410, 2001.

20. A. H. Andersen and A. C. Kak, "Simultaneous algebraic reconstruction technique (SART): a superior implementation of the art algorithm," Ultrason Imaging, vol. 6, pp. 81-94, 1984.

21. W. H. Press, B. P. Flannery, S. A. Teukolosky, and W. T. Vetterling, Numerical Recipes in $C$, Second ed. Cambridge, UK: Cambridge University Press, 1992. 